\title{
Power minimization for cooperative MIMO-OFDM systems with individual user rate constraints
}

\author{
Chih-yu Hsu*, Phee Lep Yeoh and Brian S. Krongold
}

\begin{abstract}
We propose a continuous rate and power allocation algorithm for multiuser downlink multiple-input multiple-output orthogonal frequency-division multiplexing (MIMO-OFDM) systems with coordinated multipoint (CoMP) transmission that guarantees to satisfy individual rate target across all users. The optimization problem is formulated as a total transmit power minimization problem subject to per-user rate targets and per-antenna power constraints across multiple cooperating base stations. While the per-antenna power constraint leads to a more complex optimization problem, it is a practical consideration that limits the average transmit antenna power and helps to control the resulting high peak powers in OFDM. Our proposed algorithm uses successive convex approximation (SCA) to transform the non-convex power minimization problem and dynamically allocate power to co-channel user terminals. We prove that the transformed power minimization problem is convex and that our proposed SCA algorithm converges to a solution. The proposed algorithm is compared with two alternative approaches: (1) iterative waterfilling (IWF) and (2) zero-forcing beamforming (ZFB) with semi-orthogonal user selection. Simulation results highlight that the SCA algorithm outperforms IWF and ZFB in both medium- and low-interference environments.
\end{abstract}

Keywords: MIMO-OFDM, CoMP, Power allocation, Successive convex approximation

\section{Introduction}

Intercell interference (ICI) is a limiting factor on the throughput performance of downlink multiuser multipleinput multiple-output (MIMO) orthogonal frequencydivision multiplexing (OFDM) systems. User terminals (UTs) located at the cell edge are particularly susceptible to interference from base stations (BSs) that are operating in proximity within the same frequency. In this paper, we consider the use of coordinated multipoint (CoMP) transmission with joint processing to mitigate the effect of ICI, which is a key technology in next-generation networks [1-3]. Joint processing is accomplished by sharing channel state information and user data between multiple BSs via a high-speed low-delay optical backhaul. In doing so, ICI can be mitigated by transmitting user data to a UT simultaneously from all the cooperating BSs $[4,5]$.

*Correspondence: cyhsu@student.unimelb.edu.au

Department of Electrical and Electronic Engineering, The University of

Melbourne, Melbourne, Australia
In addition to mitigating ICI using joint processing, resource allocation algorithms can be employed in conjunction with CoMP to achieve substantial improvements in multiuser MIMO system performance [6-8]. In [6], the system performance is improved by joint power allocation and linear precoding for multiuser MIMO systems with CoMP under per-antenna power constraints. In [7], the received signal-to-interference-plus-noise ratio for individual user is enhanced by adaptive nonlinear precoding and power allocation for CoMP systems with multiuser MIMO under total BS and per-BS power constraints. In [8], the joint linear precoding and power allocation for multiuser MIMO systems with CoMP are solved by convex optimization techniques under per-BS power constraints to improve the system performance.

The resource allocation problem for downlink MIMOOFDM systems has been studied extensively for the single-user case $[9,10]$. However, the optimization problem for multiuser MIMO-OFDM systems becomes

\section{Springer}

(c) $2016 \mathrm{Hsu}$ et al. Open Access This article is distributed under the terms of the Creative Commons Attribution 4.0 International License (http://creativecommons.org/licenses/by/4.0/), which permits unrestricted use, distribution, and reproduction in any medium, provided you give appropriate credit to the original author(s) and the source, provide a link to the Creative Commons license, and indicate if changes were made. 
mathematically challenging as the problem becomes nonconvex in the presence of interference. As a result, obtaining a globally optimal solution is difficult to achieve. Dirty paper coding (DPC) was first proposed in [11] to achieve broadcast channel capacity for single-cell MIMO systems, and it was extended to solve the nonconvex sum-rate maximization problem for multicell systems [12]. The DPC employs a nonlinear precoding scheme which presubtracts interference to achieve channel capacity. However, DPC requires high computational demands in successive encodings and decodings which makes it difficult to be implemented in practice.

Suboptimal strategies, such as iterative waterfilling (IWF) [13] and zero-forcing beamforming (ZFB) [14], have been proposed to solve the non-convex problem. The IWF approach in [13] treats interference as a channel noise component which transforms the optimization problem into a convex one. As a result, an equilibrium can be achieved by performing a competitive waterfillingbased algorithm iteratively across all UTs. The ZFB in [14] eliminates interference by employing zero-forcing beamformers. This allows powers to be allocated in interference-free OFDM subchannels via the waterfilling strategy. However, the performance of ZFB is limited by the number of transmit antennas and the mutual orthogonality of the UT channel gains. As a result, a semiorthogonal user selection is proposed in [15] to select a subgroup of UTs that results in the lowest mutual interference.

In this paper, we introduce a new resource allocation algorithm for multiuser downlink MIMO-OFDM systems that guarantees to satisfy a minimum rate constraint for each UT. The algorithm aims to minimize total transmit power subject to per-UT rate targets and per-antenna power constraints. Similar optimization problem has been considered in [16] for multi-cell OFDMA networks and for MIMO broadcast channels in [17]. We focus on a centralized implementation of the proposed algorithm for joint processing strategy in a multicell scenario. In doing so, we assume that perfect knowledge of all channel gains and user messages are shared via an optical backhaul which interconnects all the cooperating BSs to a central processor as shown in Fig. 1. The execution of the proposed algorithm is accomplished by allocating powers to co-channel UTs in the presence of multiuser interference (MUI) formulated as an optimization problem. As a result, the optimization problem is non-convex which is difficult to solve. To overcome this, we adopt the successive convex approximation (SCA)-based technique in [18] to transform the problem into a convex one. In [18], a SCA technique is developed for solving a non-convex dynamic spectrum management in the digital subscriber line technology with crosstalk. The algorithm attempts to jointly optimize desired signal powers and interference powers through an iterative convex approximation procedure. The same technique has been adopted to solve resource allocation problems for both single-cell MIMO-OFDMA in [19] and multicell OFDMA in [20-22] wireless networks. The SCA approach has been demonstrated in [18] to outperform the IWF algorithm. The SCA approach allows us to obtain locally optimal solutions using the dual Lagrange decomposition technique with the aid of subgradient-based methods [23].

The main contributions of the paper are summarized as follows:

- We establish an optimization approach for minimizing total transmit power while achieving per-UT rate targets. We perform eigenbeamforming on each MIMO-OFDM subchannel, with the aid of singular value decomposition, to obtain precoding and postprocessing matrices for the BS and UT, respectively.

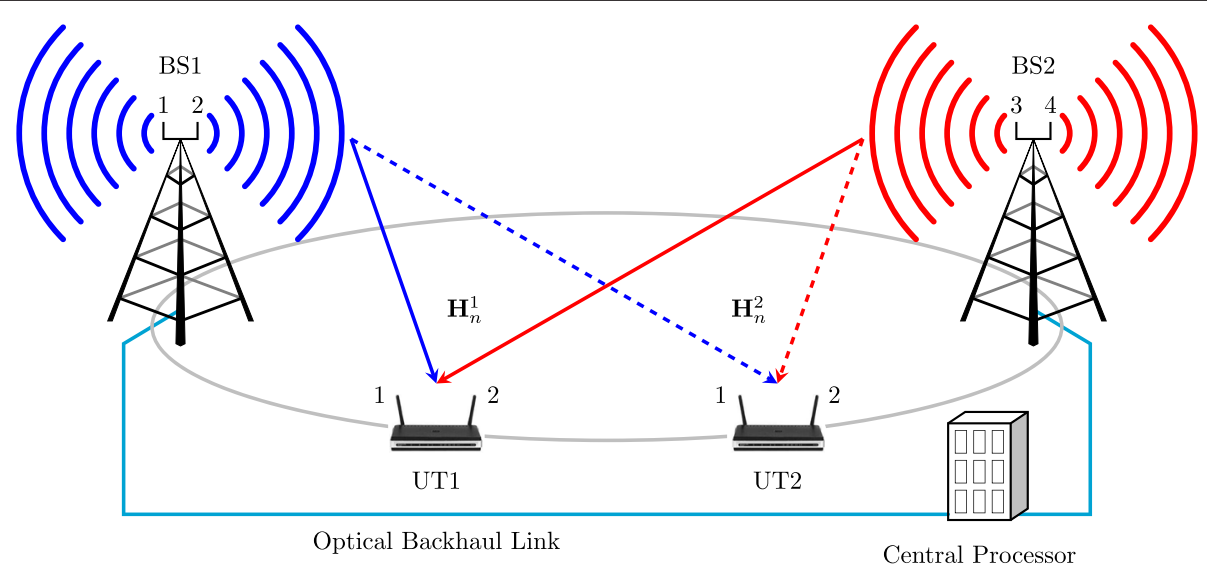

Fig. 1 A downlink MIMO-OFDM system with $M=2$ CoMP base stations transmitting to $K=2$ user terminals and $L_{T}=L_{R}=2$ 
- We derive an iterative algorithm, which is based on the SCA approach in [18], to solve the non-convex power minimization problem in which a minimum rate target is achieved for each UT. A

convex-equivalent optimization problem is obtained using the proposed iterative algorithm. In doing so, we provide a convexity proof for the transformed problem and we show that the proposed algorithm can converge to a unique solution.

- We consider the per-antenna average transmit power constraint, which limits the average transmit antenna power. As a result, the high peak power of each transmit antenna can be indirectly constrained. This ensures that the peak power is limited at an acceptable level which does not exceed the dynamic range of a high-powered amplifier, thereby causing nonlinear transmission effects. The issue of high peak powers is often overlooked in resource allocation problems which only consider a total power constraint.

- We compare our proposed algorithm with two other suboptimal algorithms IWF [24] and ZFB with semiorthogonal user selection [15]. We adopt an empirical path loss model, the COST-231 Hata empirical model [25], to model various interference environments.

A much more complicated problem would be the joint adaptive beamforming design and power allocation with a minimum mean square error receiver used to suppress the inter-user interference. While this problem tends to be intractable, our proposed algorithm could be applied on top of a coordinated beamforming method across all cooperating base stations. Furthermore, the proposed SCA algorithm is suited to fixed-wireless applications in sparsely populated regions that require high UT data rates over large network areas. A prime example is the provision of wireless broadband in rural areas where the channel gains are quasi-stationary [26]. Our algorithm is also suitable for implementation in small cells with low user mobility.

The paper is organized as follows. The system model is introduced in Section 2. The formulation of total transmit power minimization problem is presented in Section 3. The fundamental of the SCA-based algorithm is outlined in Section 4. This section also includes the convexity proof for the convex-approximated optimization problem transformed by the proposed SCA algorithm and the convergence of the proposed algorithm. Section 5 presents the numerical results of the optimization problem. Concluding remarks are presented in Section 6.

\section{System model}

In this paper, we consider a downlink multiuser MIMOOFDM system with $N$ subchannels. The system consists of $M$ cooperating BSs each with $L_{T}$ transmit antennas, as shown in Fig. 1. These BSs are interconnected by a high-speed optical backhaul for exchanging CSI and user data for joint processing. The optical backhaul is then connected to a central processor for executing a centralized implementation of our proposed power allocation algorithm, which is based on the CSI of each OFDM subchannel from the cooperating BSs. There are $K \mathrm{UT}$, each equipped with $L_{R}$ receive antennas. The spatial degree of freedom for the MIMO-OFDM system is defined as $L \leq \min \left(M L_{T}, L_{R}\right)$. We assume that perfect CSI knowledge between transmit-receive antenna pairs is known to both BSs and UTs. The CoMP configuration with joint processing operation can be envisioned as a multiuser MIMO system with distributed transmit antenna. The channel gains of these distributed transmit antennas consist various path loss profiles depending on the relative distances between the distributed transmit and receive antennas.

Assuming signals received at UTs from all cooperating BSs arrive at the same, the discrete-time complex baseband received signal in the $n$th MIMO-OFDM subchannel, denoted as $\mathbf{y}_{n}$, for all $K$ UTs after postprocessing is modeled as

$$
\mathbf{y}_{n}=\mathbf{U}_{n}^{\mathrm{H}} \mathbf{H}_{n} \mathbf{V}_{n} \mathbf{x}_{n}+\mathbf{w}_{n},
$$

where $\mathbf{H}_{n} \triangleq\left[\mathbf{H}_{n}^{1} \cdots \mathbf{H}_{n}^{K}\right]^{\mathrm{T}}$ is the complex channel gain matrix and each matrix $\mathbf{H}_{n}^{k} \in \mathbb{C}^{L_{R} \times M L_{T}}$ is independently and identically distributed (i.i.d.) random variables, each of which is drawn from a zero mean and unit variance circularly symmetric complex Gaussian distribution $\mathcal{C N}(0,1)$ in the $n$th subchannel for the $k$ th UT. The term in the $\mathbf{H}_{n}^{k}$ matrix can be interpreted as

$$
\begin{aligned}
h_{i, j}^{k}[n]= & n \text {th OFDM subchannel gain from } \operatorname{Tx} j \text { to } \\
& \operatorname{Rx} i \text { in } k \text { th UT. }
\end{aligned}
$$

We note that these subchannel gains include path attenuations, as well as both small- and large-scale fading components. The transmitted signals is denoted as $\mathbf{x}_{n} \in \mathbb{C}^{K M L_{T} \times 1}$ and the complex Gaussian noise is denoted as $\mathbf{w}_{n} \in \mathbb{C}^{K L_{R} \times 1}$ with variance $\boldsymbol{\sigma}_{n}^{2}$. The matrices $\mathbf{U}_{n}^{\mathrm{H}}=\operatorname{diag}\left(\mathbf{U}_{n}^{1 \mathrm{H}} \cdots \mathbf{U}_{n}^{K^{\mathrm{H}}}\right)$ and $\mathbf{V}_{n}=\left[\mathbf{V}_{n}^{1} \cdots \mathbf{V}_{n}^{K}\right]$ are the postprocessing and precoding matrices, respectively. Each terms $\mathbf{U}_{n}^{k} \mathrm{H}$ and $\mathbf{V}_{n}^{k}$ is obtained from the singular value decomposition (SVD) of the MIMO-OFDM subchannel matrix $\mathbf{H}_{n}^{k}$, which are given by

$$
\mathbf{H}_{n}^{k}=\mathbf{U}_{n}^{k} \Lambda_{n}^{k} \mathbf{V}_{n}^{k^{\mathrm{H}}},
$$

where $\mathbf{U}_{n}^{k} \in \mathbb{C}^{L_{R} \times L_{R}}$ and $\mathbf{V}_{n}^{k} \in \mathbb{C}^{M L_{T} \times M L_{T}}$ are the unitary transmit precoding and receiver shaping matrices, respectively, and $\boldsymbol{\Lambda}_{n}^{k} \in \mathbb{R}^{L \times M L_{T}}$ is the diagonal matrix with non-negative singular values $\sqrt{\gamma_{n, l}^{k}}, l=1, \ldots, L$ as the 
gain for the $(n, l)$ th spatial subchannel [25]. The operator $(\cdot)^{\mathrm{H}}$ represents the Hermitian transpose.

The SVD, known as the eigenbeamforming [27], is employed to decouple each MIMO-OFDM subchannel into $L$ independent parallel spatial subchannels with the singular values as the subchannel gains. This is accomplished by applying the linear transformation $\mathbf{V}_{n}^{k}$ to the transmitted symbol vector and applying the linear transformation $\mathbf{U}_{n}^{k}$ to the received symbol vector. The resulting cascaded channel can be written as

$$
\mathbf{U}_{n}^{k}{ }^{\mathrm{H}} \mathbf{H}_{n}^{k} \mathbf{V}_{n}^{k}=\mathbf{U}_{n}^{k}{ }^{\mathrm{H}} \mathbf{U}_{n}^{k} \boldsymbol{\Lambda}_{n}^{k} \mathbf{V}_{n}^{k}{ }^{\mathrm{H}} \mathbf{V}_{n}^{k}=\boldsymbol{\Lambda}_{n}^{k} .
$$

As such, a $N$-subchannel MIMO-OFDM system can be decomposed into a total of $N \times L$ spatial subchannels and with full CSI knowledge, intelligent power, and bit allocation algorithms can be employed to optimize system performance across all the spatial subchannels. The application of the eigenbeamforming does not eliminate the inter-user interference for cochannel users (like ZFB). The inter-user interference is caused by the mismatch between the $j$ th UT transmit precoding matrix and all the UT receiver shaping matrices.

Before we formulate the optimization problem, we formally define the following two signal and power domains, which will be used throughout the paper.

Definition 1. The antenna domain consists of powers that are physically transmitted by the antennas at the BSs.

Definition 2. The spatial domain consists of effective powers and signals sent in the spatial subchannels resulting from SVD.

We also define the following terms:

$$
\begin{aligned}
\tilde{R}_{n, l}^{k}= & \text { spatial rate in }(n, l) \text { th spatial subchannel for } \\
& \text { UT } k \\
\tilde{P}_{n, l}^{k}= & \text { spatial power in }(n, l) \text { th spatial subchannel for } \\
& \text { UT } k \\
P_{n, m}= & \text { transmit power in subchannel } n \text { from antenna } \\
& m,
\end{aligned}
$$

where a spatial subchannel pair is denoted by an accent with subscripts $(n, l)$ and a subchannel-antenna pair is denoted by subscripts $(n, m)$.

In the proposed power and rate allocation algorithm, we consider a continuous bit-loading scheme with a desirable rate region on the $(n, l)$ th spatial subchannel for $k$ th $\mathrm{UT}$, in bits/second/Hertz, as follows:

$$
\tilde{R}_{n, l}^{k}\left(\tilde{\mathbf{P}}_{n}\right)=\log _{2}\left[1+\operatorname{SINR}_{n, l}^{k}\left(\tilde{\mathbf{P}}_{n}\right)\right],
$$

where $\tilde{\mathbf{P}}_{n}=\left[\tilde{\mathbf{P}}_{n}^{1} \ldots \tilde{\mathbf{P}}_{n}^{K}\right]$ is the $L \times K$ spatial power matrix for the $n$th OFDM subchannel and each $\tilde{\mathbf{P}}_{n}^{K}=$
$\left[\tilde{P}_{n, 1}^{K} \ldots \tilde{P}_{n, L}^{K}\right]^{\mathrm{T}}$ is the $L \times 1$ spatial power vector for the $K$ th UT in the $n$th subchannel. The noise variance in the $(n, l)$ th spatial subchannel for the $k$ th UT is expressed as $\sigma_{n, l}^{k}$, and we assume the noise variances are constant and equal among all the spatial subchannels. The signal-tointerference-plus-noise ratio (SINR) for the $k$ th UT on the $(n, l)$ th spatial subchannel is defined as follows:

$$
\operatorname{SINR}_{n, l}^{k}\left(\tilde{\mathbf{P}}_{n}\right)=\frac{\mathrm{G}_{n, l}^{k, k} \tilde{P}_{n, l}^{k}}{\sum_{j \neq k} \mathbf{G}_{n}^{k, j}(l,:) \tilde{\mathbf{P}}_{n}^{j}+\sigma_{n, l}^{k}}, \forall l=1, \ldots, L .
$$

The term $G_{n, l}^{k, k}$ is the $(n, l)$ th spatial subchannel gain obtained from the SVD of the channel matrix. The interuser interference channel gain matrix $\mathbf{G}_{n}^{k, j}$ for the $n$th OFDM subchannel between the $k$ th UT and the $j$ th UT is defined as follows:

$$
\mathbf{G}_{n}^{k, j}(x, y)=\left|\mathbf{U}_{n}^{k}(x,:) \mathbf{H}_{n}^{k} \mathbf{V}_{n}^{j}{ }^{\mathrm{H}}(:, y)\right|^{2}, \forall x, y=1, \ldots, L .
$$

The physical interpretation of the inter-user interference gain $\mathbf{G}_{n}^{k, j}$ can be explained as the interference function from the $j$ th UT projecting onto the receiving direction of the $k$ th UT. This gives in a weighted sum of the transmitted signal in all $L$ spatial subchannels as a result of a conjugate mismatch between the transmit beamforming weights $\mathbf{V}_{n}^{j}$ and the postprocessing of $\mathbf{U}_{n}^{k}$. In the next section, we present the optimization problem that satisfy per-UT rate targets for given per-antenna transmit power constraints.

\section{Power minimization problem formulation}

The resource allocation problem in MIMO-OFDM systems can be formulated into a power minimization (PM) problem. The PM problem aims to minimize the transmit power while satisfying rate targets for each UT and transmit power constraints. For conventional rate adaptive problems, in which the objective is to maximize the total system throughput subject to a total transmit power constraint, it is intuitive that by allocating powers to the UT who has the best channel condition will maximize the overall system throughput for a given transmit power constraint. Those UTs with less favorable channel conditions will receive very little or even no data throughput as there is no rate constraint on the individual UT. In contrast, the PM problem guarantees per-UT rate targets to be satisfied while minimizing total transmit power for a given set of per-antenna power constraints.

We seek to minimize the total transmit power of a downlink multiuser MIMO-OFDM system subject to perUT target rates and per-antenna power constraints. This 
problem can be expressed mathematically as the following optimization problem:

$$
\begin{aligned}
\underset{\forall P_{n, m} \geq 0}{\operatorname{minimize}} & \sum_{m=1}^{M L_{T}} \sum_{n=1}^{N} P_{n, m} \\
\text { subject to } & \sum_{n=1}^{N} \sum_{l=1}^{L} \tilde{R}_{n, l}^{k}\left(\tilde{\mathbf{P}}_{n}, \sigma_{n, l}^{k}, \mathrm{G}_{n, l}^{k, k}, \mathbf{G}_{n}^{k, j}\right) \geq R_{\mathrm{T}}^{k} \\
& \sum_{n=1}^{N} P_{n, m} \leq P_{\max }^{m}, \forall m=1, \ldots, M L_{T}
\end{aligned}
$$

where $R_{\mathrm{T}}^{k}$ is the desirable rate target for the $k$ th UT. These rate targets must be feasible, which means there must exist a feasible power allocation such that the per-user rate target is satisfied and the per-antenna power constraints not being violated. To enhance readability, we now write $\tilde{R}_{n, l}^{k}$ without explicitly stating it being a function of $\tilde{\mathbf{P}}_{n}, \sigma_{n, l}^{k}, \mathrm{G}_{n, l}^{k, k}$ and $\mathbf{G}_{n}^{k, j}$.

We simplify the PM problem in (7) by converting the objective function and per-antenna power constraints into the spatial domain. In doing so, we derive an important relationship between spatial average powers and antenna average powers. Assuming the data symbols sent in each spatial subchannel are uncorrelated, which is expected, with zero mean and normalized to unit variance, it can be shown that, for a given subchannel $n$, the relationship between spatial and antenna powers is given by the following lemma.

Lemma 1. The relationship between antenna powers $\mathbf{P}_{n}^{k}$ and spatial powers $\tilde{\mathbf{P}}_{n}^{k}$ is given by $\mathbf{P}_{n}^{k}=\mathbf{A}_{n}^{k} \tilde{\mathbf{P}}_{n}^{k}$, where $\mathbf{A}_{n}^{k}(m, l)=\left|\mathbf{V}_{n}^{k}(m, l)\right|^{2}$.

Proof. The symbol vectors, $\tilde{\mathbf{x}}_{n}^{k} \in \mathbb{C}^{M L_{T} \times 1}$, sent in each spatial subchannel undergo a linear transformation with the precoding matrix $\mathbf{V}_{n}^{k}$ before transmission, which is given by $\mathbf{x}_{n}^{k}=\mathbf{V}_{n}^{k} \tilde{\mathbf{x}}_{n}^{k} \in \mathbb{C}^{M L_{T} \times 1}$. Furthermore, we assume these sent symbols are uncorrelated (as is expected) with zero mean and unit variance. Therefore, the relationship between antenna average powers and spatial average powers can be derived as follows:

$$
\begin{aligned}
\mathbf{P}_{n}^{k} & =\operatorname{Tr}\left\{\mathbb{E}\left[\mathbf{x}_{n}^{k} \mathbf{x}_{n}^{k^{\mathrm{H}}}\right]\right\} \\
& =\operatorname{Tr}\left\{\mathbb{E}\left[\mathbf{V}_{n}^{k} \tilde{\mathbf{x}}_{n}^{k} \tilde{\mathbf{x}}_{n}^{k \mathrm{H}} \mathbf{V}_{n}^{k^{\mathrm{H}}}\right]\right\} \\
& =\left|\left[\begin{array}{ccc}
v_{1,1}^{k} & \cdots & v_{1, M L_{T}}^{k} \\
\vdots & \ddots & \vdots \\
v_{M L_{T}, 1}^{k} & \cdots & v_{M L_{T}, M L_{T}}^{k}
\end{array}\right]\right|^{2} \mathbb{E}\left[\left|\tilde{\mathbf{x}}_{n}^{k}\right|^{2}\right] \\
& =\left|\mathbf{V}_{n}^{k}\right|^{2} \tilde{\mathbf{P}}_{n}^{k},
\end{aligned}
$$

where $\operatorname{Tr}(\cdot)$ denoted as the trace of a matrix and $|\cdot|^{2}$ denoted as the squared magnitude operation.

The term $\mathbf{A}_{n}^{k}$ refers to the power gain transformation from spatial powers to antenna powers in the $n$th subchannel for the $k$ th UT and is equal to the elementby-element squared-magnitude of the transmit precoding matrix, $\mathbf{V}_{n}^{k}$.

This relationship allows us to transform antenna powers into spatial powers which result in effective rates in each spatial subchannel. Moreover, the per-antenna power constraint prevents unbalanced power allocation among all the cooperating BSs. In the case of the total average transmit power constraint, the majority of the power would be allocated to BSs with better channel conditions. This makes the inherent peak-to-average-power ratio (PAPR) problem in the OFDM more problematic as the resulting peak transmit power at the transmit antenna may exceed the dynamic range of high-powered amplifiers (HAP) during transmission. As a result, the transmitted signal will experience nonlinear transmission effects, which compromises signal quality and, consequently, affecting the overall system performance. With per-antenna power constraints in place, the average transmit power of each antenna is constrained to a threshold in which the resulting high PAPR would not be problematic to cause irreversible nonlinear transmission effects.

The primal optimization problem in (7) is a non-convex optimization problem which the globally optimal solution is difficult to obtain. This can be shown by rewriting the per-UT rate constraint in (7) in the following expression:

$$
\begin{aligned}
\tilde{R}_{n, l}^{k}= & \log _{2}\left[G_{n, l}^{k, k} \tilde{P}_{n, l}^{k}+\sum_{j \neq k}^{K} \mathbf{G}_{n}^{k, j}(l,:) \tilde{\mathbf{P}}_{n}^{j}+\sigma_{n, l}^{k}{ }^{2}\right] \\
& -\log _{2}\left[\sum_{j \neq k}^{K} \mathbf{G}_{n}^{k, j}(l,:) \tilde{\mathbf{P}}_{n}^{j}+\sigma_{n, l}^{k^{2}}\right] .
\end{aligned}
$$

From the expression in (9), it can be seen that it is of the form of difference of concave functions (DoCF) of $\tilde{\mathbf{P}}_{n}$. Obtaining globally optimal solutions for optimization problems involve with DoCF is difficult and NP-hard [28].

\section{The proposed SCA algorithm}

To overcome the DoCF structure of the rate constraints in (7), we adopt the SCA algorithm in [18] to solve our non-convex optimization problems. The SCA algorithm converts a non-convex optimization problem into a convex one by an iterative convex approximation technique. The convex approximation is based on the following lower bound:

$$
\log _{2}(1+\operatorname{SINR}) \geq \alpha \log _{2} \operatorname{SINR}+\beta,
$$


where $\alpha$ and $\beta$ are the convex approximation constants, which dictate the accuracy of this lower bound approximation on the Pareto boundary of the achievable rate region. The approximation constants are defined as the following:

$$
\begin{aligned}
& \alpha=\frac{\operatorname{SINR}}{1+\operatorname{SINR}} \text { and } \\
& \beta=\log _{2}(1+\operatorname{SINR})-\frac{\text { SINR }}{1+\text { SINR }} \log _{2} \text { SINR. }
\end{aligned}
$$

The lower bound is improved successively by evaluating and updating $\alpha$ and $\beta$ according to (11a) and (11b) at each iteration, respectively, based on the new value $\bar{x}$. A locally optimal solution will be obtained as the lower bound converges to the actual achievable rate curve [18].

We make use of the lower bound in (10) to the per-UT rate targets and express the antenna powers in term of the spatial powers using Lemma 1 . This results in the following power minimization problem, which only involves variables in the spatial domain:

$$
\begin{aligned}
\underset{\forall \tilde{\mathbf{P}}_{n} \geq 0}{\operatorname{minimize}} & \sum_{m=1}^{M L_{T}} \sum_{k=1}^{K} \sum_{n=1}^{N} \mathbf{A}_{n}^{k}(m,:) \tilde{\mathbf{P}}_{n}^{k} \\
\text { subject to } & \sum_{n=1}^{N} \sum_{l=1}^{L} \alpha_{n, l}^{k} \log _{2}\left[\operatorname{SINR}_{n, l}^{k}\left(\tilde{\mathbf{P}}_{n}\right)\right] \\
& +\beta_{n, l}^{k} \geq R_{\mathrm{T}}^{k} \\
& \sum_{k=1}^{K} \sum_{n=1}^{N} \mathbf{A}_{n}^{k}(m,:) \tilde{\mathbf{P}}_{n}^{k} \leq P_{\max }^{m} .
\end{aligned}
$$

In order to solve this optimization problem efficiently, we adopt the Lagrange dual decomposition method [23]. First, we define the Lagrangian by converting the primal problem in (12) into an unconstrained dual optimization problem with the substitution of $\tilde{\mathbf{P}}_{n}=e^{\hat{\mathbf{P}}_{n}}$, which is given by

$$
\begin{aligned}
\mathcal{L}_{\mathrm{PM}} & \left\{\hat{\mathbf{P}}_{n}, \boldsymbol{\mu}, \lambda\right\}=\sum_{m=1}^{M L_{T}} \sum_{k=1}^{K} \sum_{n=1}^{N} \mathbf{A}_{n}^{k}(m,:) e^{\hat{\mathbf{P}}_{n}^{k}} \\
+ & \sum_{m=1}^{M L_{T}} \lambda_{m}\left[\sum_{k=1}^{K} \sum_{n=1}^{N} \mathbf{A}_{n}^{k}(m,:) e^{\hat{\mathbf{P}}_{n}^{k}}-P_{\max }^{m}\right] \\
- & \sum_{k=1}^{K} \mu_{k}\left\{\sum_{n=1}^{N} \sum_{l=1}^{L} \beta_{n, l}^{k}-R_{\mathrm{T}}^{k}+\frac{\alpha_{n, l}^{k}}{\ln 2}\left\{\ln \mathrm{G}_{n, l}^{k, k}+\hat{P}_{n, l}^{k}\right.\right. \\
& \left.\left.-\ln \left[\sum_{j \neq k}^{K} \mathbf{G}_{n}^{k, j}(l,:) e^{\hat{\mathbf{P}}_{n}^{j}}+\sigma_{n, l}^{k}{ }^{2}\right]\right\}\right\},
\end{aligned}
$$

where $\lambda=\left[\lambda_{1} \ldots \lambda_{M L_{T}}\right]$ is the $1 \times M L_{T}$ vector of Lagrange multipliers associated with each transmit antenna and $\boldsymbol{\mu}=\left[\mu_{1} \ldots \mu_{K}\right]$ is the $1 \times K$ vector of Lagrange multipliers associated with each UT rate target. The proof for the convexity of the per-UT rate target is provided in Lemma 2.

Lemma 2. The per-UT rate target in (12) is a concave function with the substitution of $\tilde{\mathbf{P}}_{n}=e^{\hat{\mathbf{P}}_{n}}$.

Proof. The per-UT rate target in (12) with the substitution of $\tilde{\mathbf{P}}_{n}=e^{\hat{\mathbf{P}}_{n}}$ is given by

$$
\begin{aligned}
\tilde{R}_{n, l}^{k}\left(e^{\hat{\mathbf{P}}_{n}}\right)= & \alpha_{n, l}^{k} \log _{2}\left[\operatorname{SINR}_{n, l}^{k}\left(e^{\hat{\mathbf{P}}_{n}}\right)\right]+\beta_{n, l}^{k} \\
= & \frac{\alpha_{n, l}^{k}}{\ln 2}\left\{\ln \mathrm{G}_{n, l}^{k, k}+\hat{P}_{n, l}^{k}\right. \\
& \left.-\ln \left[\sum_{j \neq k}^{K} \mathbf{G}_{n}^{k, j}(l,:) e^{\hat{\mathbf{P}}_{n}^{j}}+\sigma_{n, l}^{k}\right]\right\}+\beta_{n, l}^{k} .
\end{aligned}
$$

To show that the rate target in (14) is a concave function in $\hat{\mathbf{P}}_{n}$, we need to show that the Hessian of $\tilde{R}_{n, l}^{k}\left(\hat{\mathbf{P}}_{n}\right)$ is in fact a positive semi-definite matrix i.e., $\nabla^{2} \tilde{R}_{n, l}^{k}\left(\hat{\mathbf{P}}_{n}\right) \geq 0$. The Hessian of $\tilde{R}_{n, l}^{k}\left(\hat{\mathbf{P}}_{n}\right)$ is given by

$$
\nabla^{2} \tilde{R}_{n, l}^{k}\left(\hat{\mathbf{P}}_{n}\right)=\frac{1}{X^{2}}\left[X \operatorname{diag}(\mathbf{x})-\mathbf{x x}^{\mathrm{T}}\right],
$$

where the vector $\mathbf{x}$ is defined as

$$
\begin{gathered}
\mathbf{x}=\left[\mathbf{G}_{n}^{k, 1}(l,:) e^{\hat{\mathbf{P}}_{n}^{1}}, \ldots, \mathbf{G}_{n}^{k, k-1}(l,:) e^{\hat{\mathbf{P}}_{n}^{k-1}}, \mathbf{G}_{n}^{k, k+1}(l,:) e^{\hat{\mathbf{P}}_{n}^{k+1}}, \ldots,\right. \\
\left.\mathbf{G}_{n}^{k, K}(l,:) e^{\hat{\mathbf{P}}_{n}^{K}}\right],
\end{gathered}
$$

and the term $X$ is defined as

$$
X=\sum_{j=1}^{K-1} x_{j}+\sigma_{n, l}^{k^{2}} \text {. }
$$

For every $\mathbf{z} \in \mathbb{R}^{K-1}$, we have $L \triangleq \mathbf{z}^{\mathrm{T}} \nabla^{2} \tilde{R}_{n, l}^{k}\left(\hat{\mathbf{P}}_{n}\right) \mathbf{z} \geq 0$, which is given by

$$
\begin{aligned}
X^{2} L & =\mathbf{z}^{\mathrm{T}}\left[X \operatorname{diag}(\mathbf{x})-\mathbf{x x}^{\mathrm{T}}\right] \mathbf{z} \\
& =\left(\sum_{j=1}^{K-1} z_{j}^{2} x_{j}\right)\left(\sum_{j=1}^{K-1} x_{j}+{\sigma_{n, l}^{k}}^{2}\right)-\left(\sum_{j=1}^{K-1} z_{j} x_{j}\right)^{2} \\
& \geq 0,
\end{aligned}
$$

and since $\sigma_{n, l}^{k}$ is non-negative and therefore, the CauchySchwarz inequality holds [23]. 
The dual problem is then given by

$$
\underset{\mu, \lambda \succ 0}{\operatorname{maximize}} d(\boldsymbol{\mu}, \lambda)
$$

where the Lagrange dual objective function, denoted as $d(\boldsymbol{\mu}, \boldsymbol{\lambda})$, is defined as

$$
d(\boldsymbol{\mu}, \lambda)=\min _{\hat{\mathbf{P}}_{n} \succeq 0} \mathcal{L}_{P M}\left\{\hat{\mathbf{P}}_{n}, \boldsymbol{\mu}, \lambda\right\}
$$

The optimal solution of the dual problem is given by

$$
D^{*}=d\left(\boldsymbol{\mu}^{*}, \lambda^{*}\right),
$$

where $\mu^{*}$ and $\lambda^{*}$ are the optimal Lagrange multipliers. From (13) and (20), $d(\boldsymbol{\mu}, \boldsymbol{\lambda})$ is a convex function as it is a pointwise minimum of a series of weighted affine functions of $\boldsymbol{\mu}$ and $\lambda$ [23]. Therefore, the optimal Lagrange multipliers of $\boldsymbol{\mu}^{*}$ and $\lambda^{*}$, in which maximize $d(\boldsymbol{\mu}, \lambda)$, can be obtained by using the standard convex optimization techniques [23]. The corresponding optimal value of the dual problem $D^{*}$ is the lower bound for the optimal value of the approximated primal problem in (12), denoted as $P^{*}$, given by

$$
P^{*} \geq D^{*} \text {. }
$$

Since the SCA technique is employed to transform the original optimization problem in (7) into a convex one and the feasible set has a non-empty interior, the duality gap between $P^{*}$ and $D^{*}$ is in fact zero. This is due to the fact that any finite rate target is achievable for any given arbitrary large transmit powers. As a result, the approximated optimization problem in (12) satisfies the Slater's condition which implies that the strong duality condition holds [23]. Therefore, $P^{*}$ can be found by first minimizing the Lagrangian $\mathcal{L}_{\mathrm{PM}}$ in (13) to evaluate the dual objective function $d(\boldsymbol{\mu}, \boldsymbol{\lambda})$ in (20) and then maximizing $d(\boldsymbol{\mu}, \boldsymbol{\lambda})$ over all non-negative values of $\boldsymbol{\mu}$ and $\lambda$. Furthermore, the Lagrangian in (13) can be simplified into $N K$-independent subproblems using standard dual decomposition method for a given $\mu$ and $\lambda$, which is given by

$$
\mathcal{L}_{\mathrm{PM}}(\boldsymbol{\mu}, \boldsymbol{\lambda})=\sum_{n=1}^{N} \sum_{k=1}^{K} \breve{g}_{n}^{k}(\boldsymbol{\mu}, \boldsymbol{\lambda})-\sum_{m=1}^{M L_{T}} \lambda_{m} P_{\max }^{m}+\sum_{k=1}^{K} \mu_{k} R_{\mathrm{T}}^{k},
$$

where $\breve{g}_{n}^{k}$ is given by

$$
\begin{aligned}
& \breve{g}_{n}^{k}(\boldsymbol{\mu}, \lambda)=\underset{\hat{\mathbf{P}}_{n}^{k} \geq 0}{\operatorname{minimize}} \sum_{m=1}^{M L_{T}} \mathbf{A}_{n}^{k}(m,:) e^{\hat{\mathbf{P}}_{n}^{k}}\left(1+\lambda_{m}\right) \\
&-\sum_{l=1}^{L} \frac{\mu_{k} \alpha_{n, l}^{k}}{\ln 2}\left\{\ln G_{n, l}^{k, k}+\hat{P}_{n, l}^{k}-\ln \left[\sum_{j \neq k}^{K} \mathbf{G}_{n}^{k, j}(l,:) e^{\hat{\mathbf{P}}_{n}^{j}}\right.\right. \\
&\left.\left.\quad+\sigma_{n, l}^{k^{2}}\right]+\frac{\beta_{n, l} \ln 2}{\alpha_{n, l}^{k}}\right\} .
\end{aligned}
$$

This indicates that the dual problem can be solved by optimizing $N$-independent dual subproblems, each for $\breve{g}_{n}^{k}(\boldsymbol{\mu}, \lambda), \forall k=1, \ldots, K$. As a result, the overall implementation cost can be reduced significantly if the same procedure is executed repeatedly for solving each subproblem, or alternatively, $K$ parallel processors can be adopted for solving $N$ dual subproblems simultaneously to improve the convergence time of the algorithm.

For the $n$th OFDM subchannel of the $k$ th UT, the minimization in (24) over $\hat{\mathbf{P}}_{n}^{k}$ is a convex optimization problem. Therefore, the optimal value $\hat{\mathbf{P}}_{n}^{k^{*}}$ must satisfy the following Karush-Kuhn-Tucker (KKT) necessary conditions [23] simultaneously, which are given by

$$
\begin{aligned}
1-\frac{\ln 2 \mathbf{A}_{n}^{k}(m,:) e^{\hat{\mathbf{P}}_{n}^{k^{*}}}\left(1+\lambda_{m}\right)}{\mu_{k} \alpha_{n, l}^{k}}-\frac{\sum_{j \neq k} \mathbf{G}_{n}^{k, j}(l,:) e^{\hat{\mathbf{P}}_{n}^{j}}}{\sum_{j \neq k} \mathbf{G}_{n}^{k, j}(l,:) e^{\hat{\mathbf{P}}_{n}^{j}}+\sigma_{n, l}^{k}} & =0, \forall k, n, l \\
\lambda_{m}\left[\sum_{k=1}^{K} \sum_{n=1}^{N} \mathbf{A}_{n}^{k}(m,:) e^{\hat{\mathbf{P}}_{n}^{k^{*}}}-P_{\max }^{m}\right] & =0, \forall m \\
\mu_{k}\left\{R_{\mathrm{T}}^{k}-\sum_{n=1}^{N} \sum_{l=1}^{L} \alpha_{n, l}^{k} \log _{2}\left[\operatorname{SINR}_{n, l}^{k}\left(e^{\hat{\mathbf{P}}_{n}^{k^{*}}}\right)\right]+\beta_{n, l}^{k}\right\} & =0, \forall k \\
\lambda_{m} & \geq 0, \forall m \\
\mu_{k} & \geq 0, \forall k
\end{aligned}
$$

From the stationarity of the KKT conditions in (25), the optimal power allocation $\tilde{P}_{n, l}^{k}$ can be obtained by substituting $\hat{\mathbf{P}}_{n}=\ln \tilde{\mathbf{P}}_{n}$ with fixed $\boldsymbol{\lambda}$ and $\boldsymbol{\mu}$, which results in

$$
\tilde{P}_{n, l}^{k}=\frac{\mu_{k} \alpha_{n, l}^{k}}{\ln 2(\mathbf{1}+\lambda) \mathbf{A}_{n}^{k}(:, l)+\sum_{j \neq k} \mathbf{G}_{n}^{j, k}(l,:) \boldsymbol{\alpha}_{n}^{j} \mu_{j} \frac{\operatorname{SINR}_{n, l}^{j}\left(\tilde{\mathbf{P}}_{n}\right)}{G_{n, l}^{j, j} \tilde{P}_{n, l}^{j}}},
$$

where $\mathbf{1}$ is the $1 \times M L_{T}$ vector of ones and $\boldsymbol{\alpha}_{n}^{j}=$ $\left[\alpha_{n, 1}^{j} \ldots \alpha_{n, L}^{j}\right]^{\mathrm{T}}$ is the $L \times 1$ convex approximation constant vector for the $n$th OFDM subchannel of the $j$ th UT. We note that the term $\mathbf{G}_{n}^{j, k}(l,:)$ quantifies the impact of allocating $\tilde{P}_{n, l}^{k}$ to the $k$ th UT on all other UTs, which results in an altruistic approach of allocating powers to UTs that have the minimal mutual interference. This differs from the egoistic approach of IWF by maximizing the signal-tonoise ratio without regard to resulting mutual interference to all UTs.

The power allocation strategy in (26) is a standard interference function which is guaranteed to coverage to a unique solution [29]. To demonstrate this, we apply Yates' definition of standard interference function in [29] to (26) which is introduced in the following definition. 
Definition 3. An interference function $\mathcal{I}(\mathbf{p})$ is standard if for all $\mathbf{p} \succeq 0$ and the following properties are satisfied.

- Positivity: $\mathcal{I}(\mathbf{p})>0$

- Monotonicity: If $\mathbf{p} \geq \mathbf{p}^{\prime}$, then $\mathcal{I}(\mathbf{p}) \geq \mathcal{I}\left(\mathbf{p}^{\prime}\right)$

- Scalability: For all $\theta>1, \theta \mathcal{I}(\mathbf{p})>\mathcal{I}(\theta \mathbf{p})$

Lemma 3. The power allocation strategy in (26) is a standard interference function [18].

Proof. We rewrite the power allocation in (26) as

$$
\mathcal{I}_{n, l}^{k}(\tilde{\mathbf{P}})=\frac{\mu_{k} \alpha_{n, l}^{k}}{\ln 2(\mathbf{1}+\lambda) \mathbf{A}_{n}^{k}(:, l)+\sum_{j \neq k} \frac{\mathbf{G}_{n}^{j, k}(l,:) \alpha_{n}^{j} \mu_{j}}{\mathbf{G}_{n}^{k, j}(l,:) \tilde{\mathbf{P}}_{n}^{j}+\sigma_{n, l}^{k}}} .
$$

To show the power allocation in (27) is unique and it can converge to a locally optimal solution, we apply Yates' definition of standard interference function in Definition 3.

- Positivity: This follows from the fact that each term in $\mathcal{I}_{n, l}^{k}(\tilde{\mathbf{P}})$ in (27) is non-negative.

- Monotonicity: Suppose $\tilde{\mathbf{P}} \geq \tilde{\mathbf{P}}^{\prime}$, the monotonicity property follows from

$$
\begin{aligned}
\mathcal{I}_{n, l}^{k}(\tilde{\mathbf{P}}) & =\frac{\mu_{k} \alpha_{n, l}^{k}}{\ln 2(\mathbf{1}+\lambda) \mathbf{A}_{n}^{k}(:, l)+\sum_{j \neq k} \frac{\mathbf{G}_{n}^{j, k}(l,:) \boldsymbol{\mathbf { \alpha }}_{n}^{j} \mu_{j}}{\mathbf{G}_{n}^{k, j}(l,) \tilde{\mathbf{P}}_{n}^{j}+\sigma_{n, l}^{k}}} \\
& \geq \frac{\mu_{k} \alpha_{n, l}^{k}}{\ln 2(\mathbf{1}+\lambda) \mathbf{A}_{n}^{k}(:, l)+\sum_{j \neq k} \frac{\mathbf{G}_{n}^{j, k}(l,:) \alpha_{n}^{j} \mu_{j}}{\mathbf{G}_{n}^{k, j}(l,:) \tilde{\mathbf{P}}_{n}^{j^{\prime}}+\sigma_{n, l}^{k}{ }^{2}}} \\
& =\mathcal{I}_{n, l}^{k}\left(\tilde{\mathbf{P}}^{\prime}\right)
\end{aligned}
$$

- Scalability: Suppose $\tilde{\mathbf{P}}=\theta \tilde{\mathbf{P}}^{\prime}$ for $\theta>1$, the scalability property follows from

$$
\begin{aligned}
\theta \mathcal{I}_{n, l}^{k}(\tilde{\mathbf{P}}) & =\frac{\mu_{k} \alpha_{n, l}^{k}}{\frac{1}{\theta} \ln 2(\mathbf{1}+\lambda) \mathbf{A}_{n}^{k}(:, l)+\frac{1}{\theta} \sum_{j \neq k} \frac{\mathbf{G}_{n}^{j, k}(l,:) \boldsymbol{\alpha}_{n}^{j} \mu_{j}}{\mathbf{G}_{n}^{k, j}(l,:) \tilde{\mathbf{P}}_{n}^{j}+\sigma_{n, l}^{k}{ }^{2}}} \\
& >\frac{\mu_{k} \alpha_{n, l}^{k}}{\ln 2(\mathbf{1}+\lambda) \mathbf{A}_{n}^{k}(:, l)+\sum_{j \neq k} \frac{\mathbf{G}_{n}^{j, k}(l, ;) \boldsymbol{\alpha}_{n}^{j} \mu_{j}}{\mathbf{G}_{n}^{k, j}(l,:) \theta \tilde{\mathbf{P}}_{n}^{\tilde{P}^{\prime}}+\sigma_{n, l}^{k}{ }^{2}}} \\
& =\mathcal{I}_{n, l}^{k}\left(\theta \tilde{\mathbf{P}}^{\prime}\right)
\end{aligned}
$$

The final step is to find $\mu^{*}$ and $\lambda^{*}$ that maximize $d(\mu, \lambda)$ over all $\mu \succeq 0$ and $\lambda \succeq 0$. This is accomplished by a gradient descent method [23] which is given by the following: $\lambda_{m}{ }^{[s+1]}=\left[\lambda_{m}{ }^{[s]}+v\left(\sum_{k=1}^{K} \sum_{n=1}^{N} \mathbf{A}_{n}^{k}(m,:) \tilde{\mathbf{P}}_{n}^{k^{[s+1]}}-P_{\max }^{m}\right)\right]^{+}$

$\mu_{k}^{[s+1]}=\left[\mu_{k}^{[s]}+\epsilon\left(R_{\mathrm{T}}^{k}-\sum_{n=1}^{N} \sum_{l=1}^{L} \tilde{R}_{n, l}^{k}{ }^{[s+1]}\right)\right]^{+}$,

respectively, for some fixed $\tilde{\mathbf{P}}_{n}^{k}$, where $\epsilon$ and $v$ are step sizes for each iteration, and $s$ is the iteration number. The updated Lagrange multipliers $\boldsymbol{\mu}^{[s+1]}$ and $\lambda^{[s+1]}$ are then substituted back into (26) to obtain the new power allocation $\tilde{\mathbf{P}}_{n}^{k^{[s+1]}}$, and the resulting rate allocation $\tilde{R}_{n, l}^{k}{ }^{[s+1]}$ is obtained from $\tilde{\mathbf{P}}_{n}^{k^{[s+1]}}$ using (4). The iterative procedure terminates when the duality gap between the primal and dual objective function approaches to zero. The PMSCA algorithm is outlined in Algorithm 1. We initialize the algorithm with a high-SINR approximation with $\alpha=1$ and $\boldsymbol{\beta}=0$ [18]. Before we present numerical results in next section, we introduce IWF and ZFB with semiorthogonal user selection, which we used to compare the performance of our proposed algorithm.

\subsection{IWF}

In IWF, the power allocation for each MIMO-OFDM subchannel is performed by assuming that the inter-user interference is constant and treating it as a part of channel noise. As a result, the original nonconvex optimization problem is transformed into a convex one. An equilibrium is achieved by performing the waterfilling solution iteratively across all the UTs in the system. In numerical simulations, we first perform a SVD on individual MIMOOFDM subchannel to obtain the individual subchannel gains. These subchannel gains are then used to perform

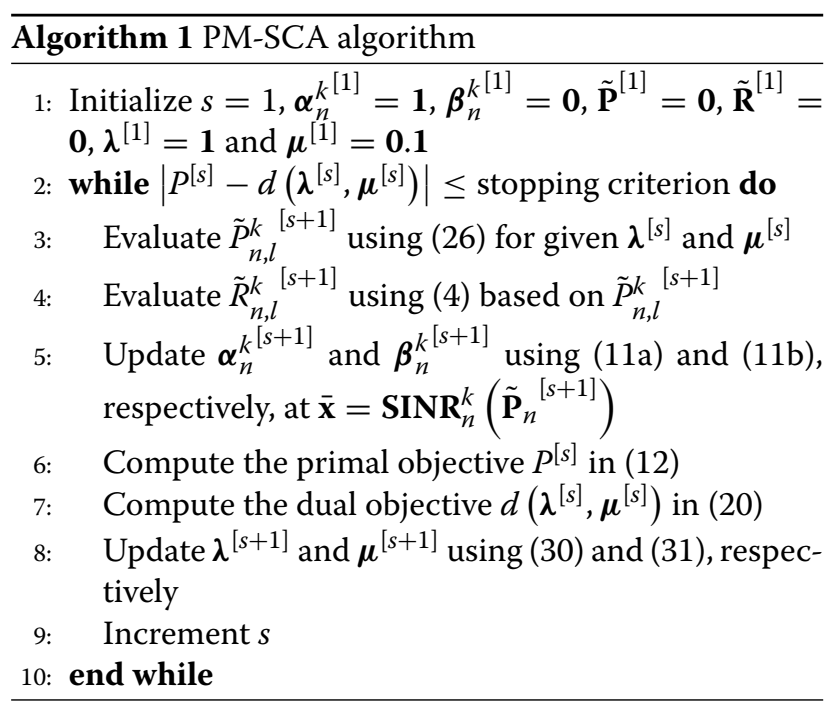


the power allocation, which is based on the iterative waterfilling algorithm across all the UTs.

\subsection{ZFB with semi-orthogonal user selection}

In ZFB, orthogonal beamformers are used to eliminate the inter-user interference for co-channel UTs. This transforms the original nonconvex optimization problem into a convex one, and the waterfilling algorithm is performed across MIMO-OFDM subchannels to obtain a suboptimal solution. However, an efficient user selection algorithm is needed for finding co-channel UTs with less mutual interference in order to maximize the system performance, in particular, when the number of UTs is large. Therefore, a semi-orthogonal user selection is introduced for effectively finding near-orthogonal co-channel UTs to occupy the limited number of zero-forcing beamformers, which is governed by the number of transmit antennas.

\section{Simulation results and discussion}

In this section, we present the numerical results to evaluate our proposed algorithm against IWF and ZFB with semi-orthogonal user selection. We consider a downlink fixed-wireless MIMO-OFDM system with $N=32$ OFDM subchannels and $M=3 \mathrm{BSs}$ where each $\mathrm{BS}$ is equipped with $L_{T}=2$ transmit antennas. All the cooperating BSs are $d \mathrm{~km}$ separated from each other. These BSs are assumed to be interconnected by an optical backhaul at which channel gains and user data are shared between cooperating BSs for joint processing. The proposed algorithm is executed centrally by a central processor which is also connected to BSs via the backhaul. We focus on $K=5$ UTs in the simulation, and each UT is equipped with $L_{R}=$ 2 receive antennas. As shown in Fig. 2, the UTs are randomly distributed in a virtual radius of $r=100 \mathrm{~m}$ which is located between the two cooperating BSs to simulate a cell-edge environment. The COST-231 Hata empirical model [25] is used for predicting the path loss of the channels in rural (flat) environments for typical macrocell deployments. The transmission loss, $L_{d}$, expressed in decibels is given by [25]

$$
\begin{aligned}
L_{d}= & 46.3+33.9 \log _{10} f-13.82 \log _{10} h_{t}-a\left(h_{r}\right) \\
& +\left(44.9-6.55 \log _{10} h_{t}\right) \log _{10} d+C_{m},
\end{aligned}
$$

where $f$ is the carrier frequency in $\mathrm{MHz}, d$ is the distance between BS and UT antennas in $\mathrm{km}$, and $h_{t}$ is the height of the BS above ground level in $\mathrm{m}$. The parameter $C_{m}$ is defined as $0 \mathrm{~dB}$ for suburban or rural environments and $3 \mathrm{~dB}$ for metropolitan environments. The parameter $a\left(h_{r}\right)$ is defined for rural environments as [26]

$$
a\left(h_{r}\right)=\left(1.1 \log _{10} f-0.7\right) h_{r}-\left(1.56 \log _{10} f-0.8\right),
$$

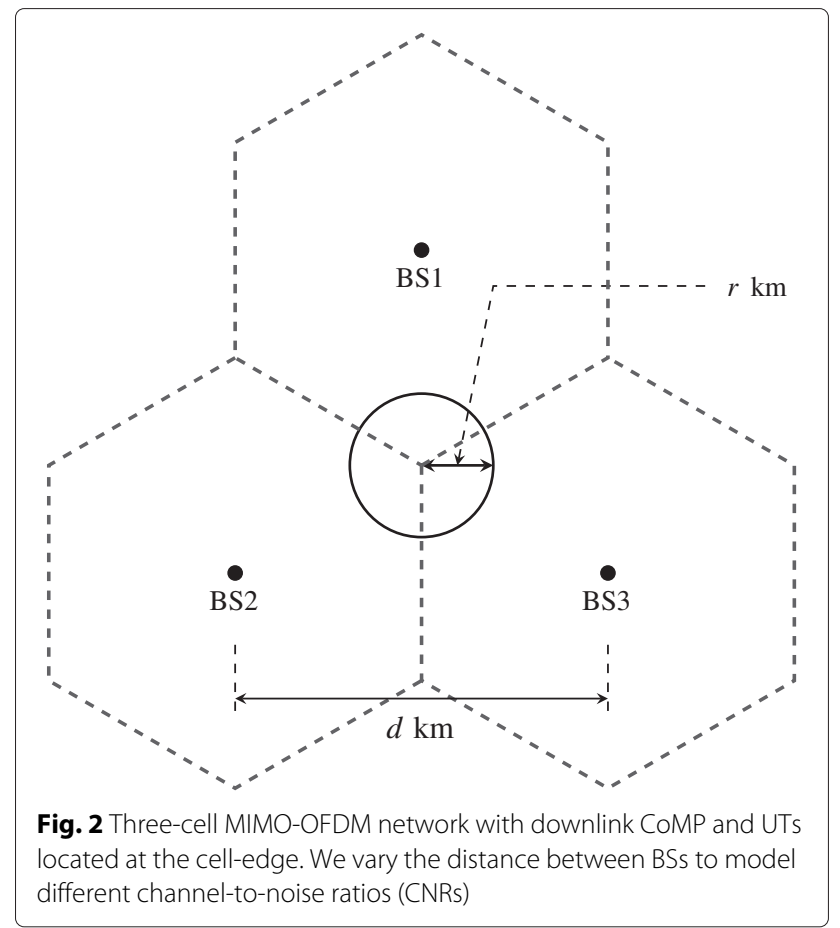

where $h_{r}$ is the height of the UT above ground level in meters. The simulation parameters from $[25,30,31]$ are given in Table 1.

In the simulation results, we investigate the power minimization performance of our proposed algorithm in various interference environments by varying the distance between cooperating BSs, which is denoted as $d$ in Fig. 2, ranging from 5 to $40 \mathrm{~km}$ in a typical LTE macrocell deployment [31]. Based on these distances, a received

Table 1 COST 231 path-loss model parameters [25, 30, 31]

\begin{tabular}{lc}
\hline Simulation parameters & 10 \\
\hline Bandwidth (MHz) & 2 \\
Carrier frequency $(\mathrm{GHz})$ & 30 \\
BS height $(\mathrm{m})$ & 46 \\
Maximum Tx power (dBm) & 2 \\
RF feeder cable/connector loss (dB) & 18 \\
Antenna gain $(\mathrm{dBi})$ & 5 \\
Receiver height $(\mathrm{m})$ & 8 \\
Antenna gain $(\mathrm{dBi})$ & 7 \\
Noise figure $(\mathrm{dB})$ & -174 \\
Thermal noise $(\mathrm{dBm} / \mathrm{Hz})$ & -97 \\
Receiver noise floor $(\mathrm{dBm})$ & 8 \\
Slow fading margin $(\mathrm{dB})$ & \\
\hline
\end{tabular}


channel-to-noise ratio (CNR) on the $(n, l)$ th spatial subchannel for the $k$ th UT, which is given by [10]

$$
\mathrm{CNR}_{n, l}^{k}=\frac{\Lambda_{n, l}^{k}}{\sigma_{n, l}^{k^{2}}},
$$

where $\Lambda_{n, l}^{k}$ is the effective channel gain after precoding and postprocessing. The noise power is assumed to be equal across all OFDM subchannels. We average the simulation results over a total of 16,000 channel realizations, which is obtained from 100 simulation iterations for each UT subchannel.

Figure 3 shows total power minimization comparison between our proposed SCA and other two alternative approaches of IWF in [24] and ZFB with semi-orthogonal user selection [15]. The BS-to-BS separation distance is set to $d=5 \mathrm{~km}$ and the resulting average received $\mathrm{CNR}=-7.66 \mathrm{~dB}$ which indicates in a low-interference environment where the interference power is insignificant compared with the noise power. The results show that the proposed SCA algorithm provides the lowest total transmit power compared with IWF and ZFB for a given per-UT rate target. We notice that IWF provides a similar performance to SCA whereas ZFB results in the highest total transmit power for a given per-UT target rate. For example, SCA and IWF offer an approximately $50 \mathrm{~W}$ of saving in total transmit power to achieve a per-UT rate target of $4.5 \mathrm{Mbits} / \mathrm{s}$.

Figure 4 compares SCA, IWF, and ZFB with a distance between cooperating BSs of $d=20 \mathrm{~km}$. The resulting average received $\mathrm{CNR}=2.94 \mathrm{~dB}$ which indicates a medium-interference environment where the interference power is comparable to the noise power. In this scenario, we notice that our proposed SCA algorithm can achieve the lowest total transmit power for a given per-UT rate target. For a total transmit power of $120 \mathrm{~W}$ between cooperating BSs, we can see that IWF is limited to $19 \mathrm{Mbits} / \mathrm{s}$ per UT as a result of interference. Despite the canceling of interference between the scheduled UTs, we see that ZFB results in a higher total transmit power of $102 \mathrm{~W}$ compared to SCA of $67 \mathrm{~W}$ for a rate target of $24 \mathrm{Mbits} / \mathrm{s}$ per UT. This is due to the reduction in the effective channel gain of the scheduled UTs as a result of performing ZFB on the channel gain matrices of the scheduled UTs in each subchannel.

Figure 5 compares SCA, IWF, and ZFB with the BSto-BS separation distance set to $d=40 \mathrm{~km}$. The average received $C N R=21.12 \mathrm{~dB}$, which models a highinterference environment where the noise power is insignificant compared to the interference power. The plot shows that ZFB results in the lowest total transmit power for a given per-UT target rate compared with SCA and IWF. This is because ZFB cancels interferences between scheduled UTs which are selected by the semi-orthogonal user selection in each subchannel. For a total power constraint across the two cooperating BSs, we see that SCA and IWF can achieve a maximum of 45 and $25 \mathrm{Mbits} / \mathrm{s}$ per UT, respectively. From this comparison, we notice that SCA offers a better interference management than IWF with a lower total transmit power for a given per-UT rate target.

Next, we investigate the relationship between the minimum achievable rates per-UT and the coverage radius, $r$. To ensure the distribution of UT changes with the

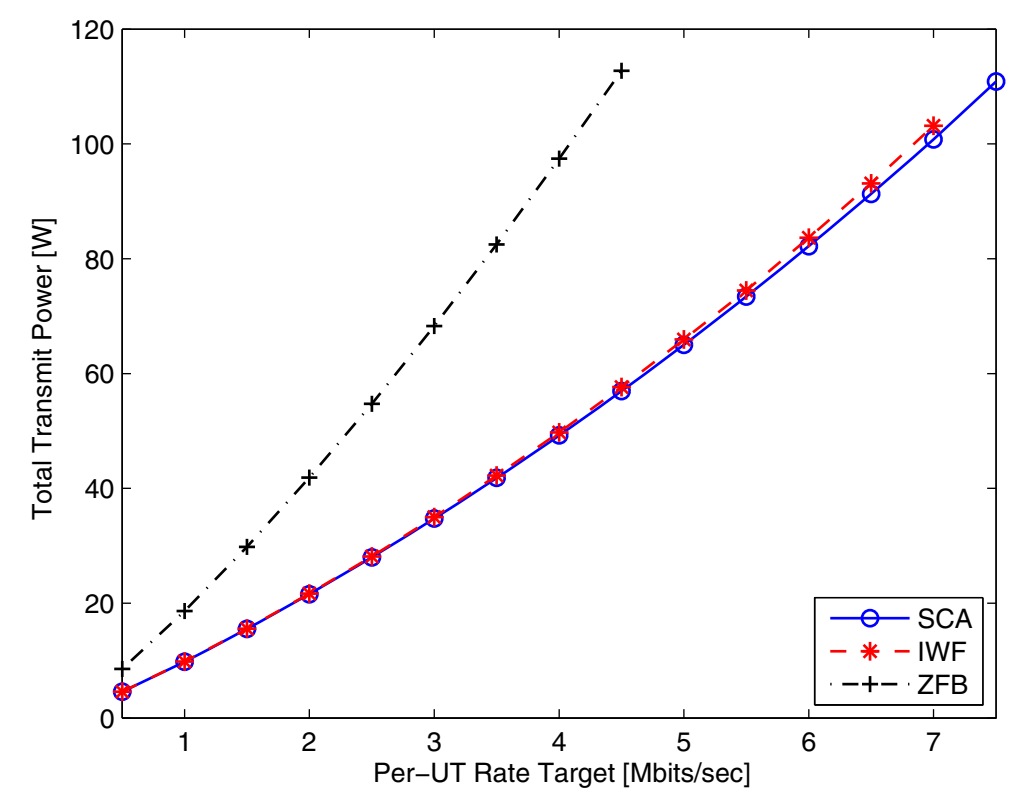

Fig. 3 Transmit power comparison of SCA, IWF, and ZFB in low-interference environments with different per-UT rate targets and $d=40 \mathrm{~km}$ 


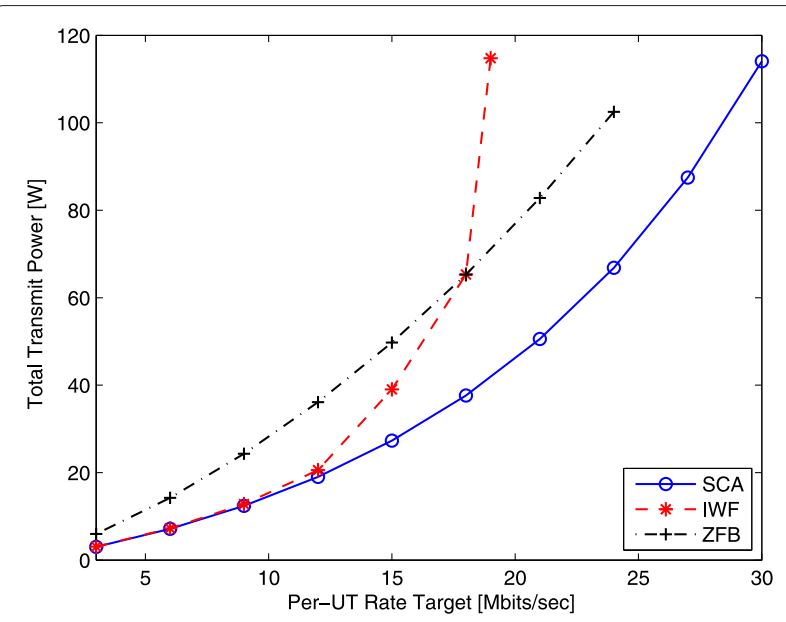

Fig. 4 Transmit power comparison of SCA, IWF, and ZFB in medium-interference environments with different per-UT rate targets and $d=20 \mathrm{~km}$

coverage radius, we place the UTs uniformly distributed on the circumference of the coverage circle as the circle expands to simulate UTs scatter between cooperating BSs. The minimum achievable rates refer to the minimum rate between per-UT rate targets when the per-antenna powers are close to be fully utilized. The results are obtained from the average of 100 simulation iterations.

Figure 6 compares the minimum achievable rate of SCA, IWF, and ZFB with a BS-to-BS separation distance $d=$ $40 \mathrm{~km}$. In this scenario, we notice that both SCA and IWF result in a similar performance whereas ZFB achieves the lowest minimum per-UT rate target. Comparing SCA and IWF, the minimum per-UT rate target increases with coverage radius as the interference decreases.

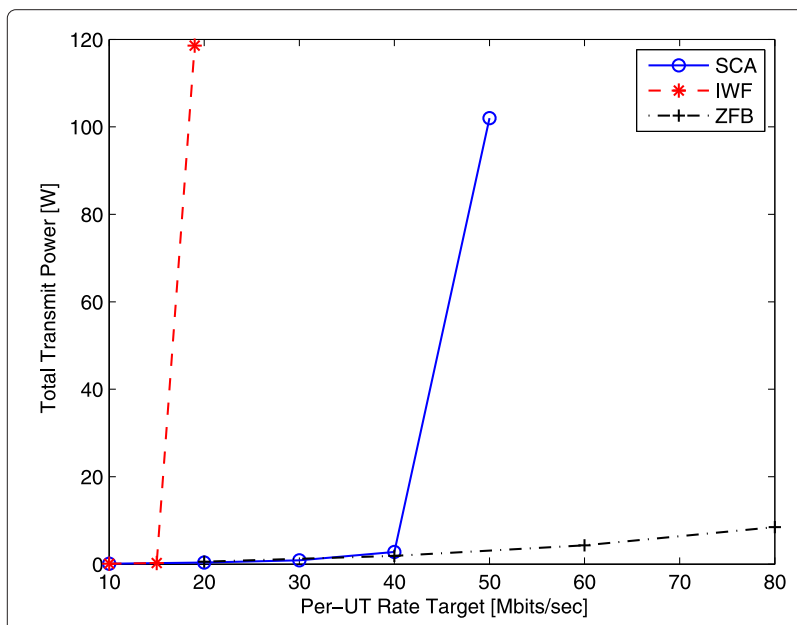

Fig. 5 Transmit power comparison of SCA, IWF, and ZFB in high-interference environments with different per-UT rate targets and $d=5 \mathrm{~km}$
Figure 7 compares the minimum achievable rate of SCA, IWF, and ZFB with a BS-to-BS separation distance $d=$ $20 \mathrm{~km}$. The performance between SCA and IWF widens as the interference increases at each coverage radius. ZFB outperforms IWF when the coverage radius is less than $4.5 \mathrm{~km}$ as the MUI is the dominating factor in system performance. The altruistic approach of allocating power in SCA is able to outperform ZFB despite the MUI completely eliminated by the beamformers.

Figure 8 compares the minimum achievable rate of SCA, IWF, and ZFB with a BS-to-BS separation distance $d=$ $5 \mathrm{~km}$. In this scenario, the performance of both SCA and IWF is limited by the severity of the MUI and the minimum achievable rates are 48 and $20 \mathrm{Mbit} / \mathrm{s}$, respectively. As expected, ZFB results in the best performance as the approach provides interference-free MIMO-OFDM spatial subchannels for scheduled UTs. From these plots, we notice that the performance gap between SCA and IWF depends on the severity of the MUI. The egoistic approach of allocating powers in IWF results in lower performance compared to the interference minimizing approach of SCA.

\subsection{Complexity analysis}

The computational complexity of the proposed algorithm, IWF and ZFB, consists of two stages: (1) computation of beamformers for each MIMO-OFDM subchannel and (2) updates of power allocation and Lagrange multipliers. We focus on the computational complexity of obtaining beamformers as the power and Lagrange multipliers update has fixed complexity and is negligible compared to the beamforming of each MIMO-OFDM subchannel. The computational complexity of each algorithm is calculated as follows:

- For the proposed algorithm: The eigenbeamforming of each MIMO-OFDM subchannel is obtained by the SVD of MIMO-OFDM subchannel $\mathbf{H}_{n}^{k}$. The channel matrix $\mathbf{H}_{n}^{k}$ is a $L_{R} \times M L_{T}$ complex matrix. To obtain the SVD of each $\mathbf{H}_{n}^{k}$ requires $8\left(4 L_{R}^{2} M L_{T}+8 L_{R}\right.$ $\left.\left(M L_{T}\right)^{2}+9\left(M L_{T}\right)^{3}\right)$ complex floating point operations [32]. The total number of complex floating point operations across all MIMO-OFDM subchannel and UTs is approximately

$$
\sum_{k=1}^{K} \sum_{n=1}^{N} 8 k n\left[4 L_{R}^{2} M L_{T}+8 L_{R}\left(M L_{T}\right)^{2}+9\left(M L_{T}\right)^{3}\right] .
$$

Therefore, the overall computational complexity of the proposed algorithm is

$$
\mathcal{O}\left\{8 K N\left[4 L_{R}^{2} M L_{T}+8 L_{R}\left(M L_{T}\right)^{2}+9\left(M L_{T}\right)^{3}\right]\right\} .
$$




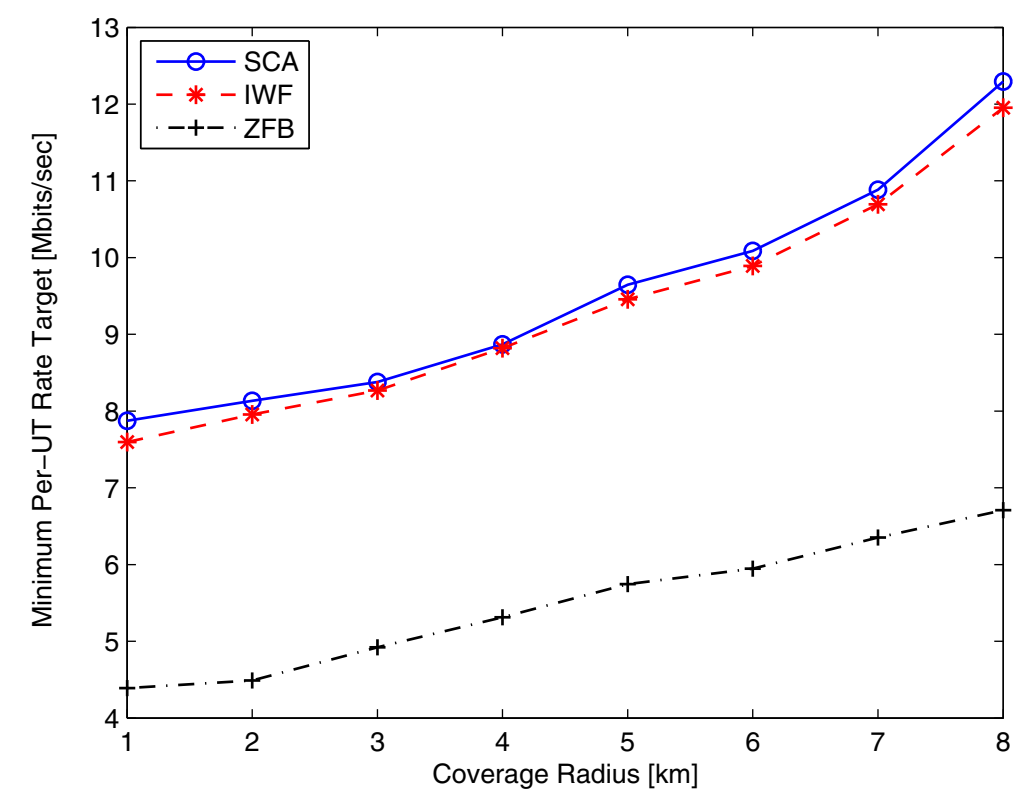

Fig. 6 Minimum achievable rate comparison of SCA, IWF, and ZFB with a BS-to-BS separation of $d=40 \mathrm{~km}$

- The overall computational complexity of IWF is approximately the same as the proposed algorithm since the eigenbeamforming is performed across all MIMO-OFDM subchannels, which is given by

$$
\mathcal{O}\left\{8 K N\left[4 L_{R}^{2} M L_{T}+8 L_{R}\left(M L_{T}\right)^{2}+9\left(M L_{T}\right)^{3}\right]\right\} .
$$

- For ZFB with semi-orthogonal user selection: This algorithm consists of two stages: (1) semi-orthogonal user selection and (2) obtaining zero-forcing beamformers. The computational complexity of semi-orthogonal user selection is given by $\mathcal{O}\left[K N\left(L_{T}\right)^{3}\right]$ [33]. Finding zero-forcing beamformers involves block diagonalization across all the

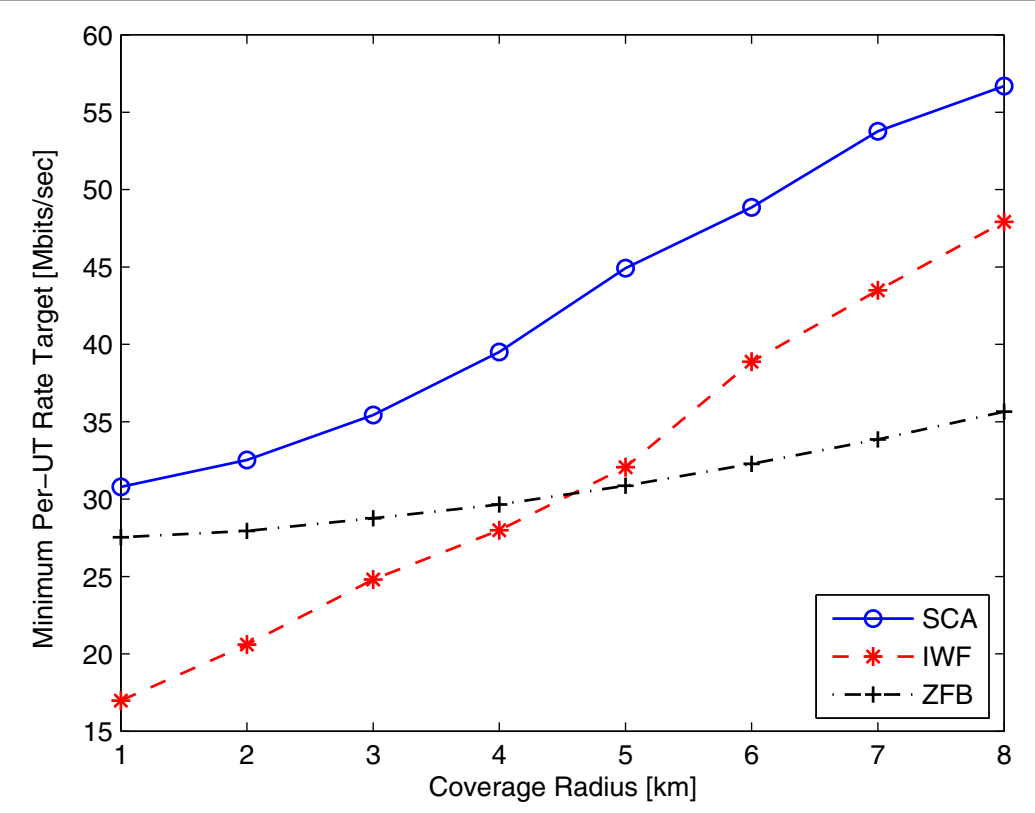

Fig. 7 Minimum achievable rate comparison of SCA, IWF, and ZFB with a BS-to-BS separation of $d=20 \mathrm{~km}$ 


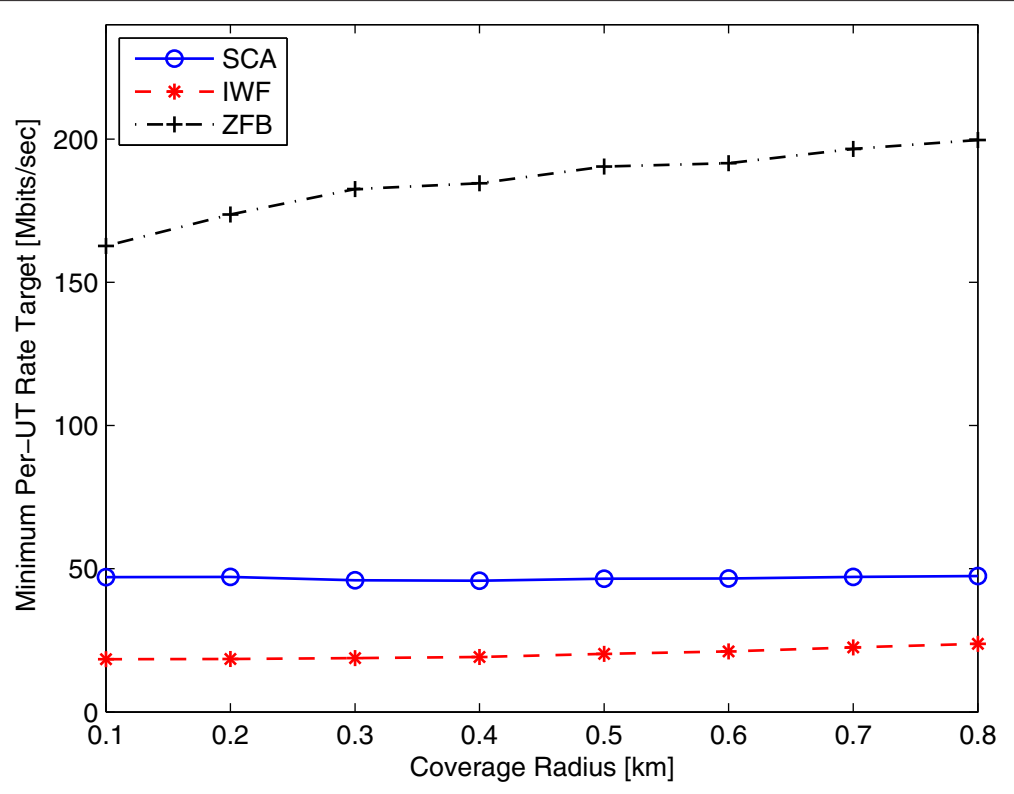

Fig. 8 Minimum achievable rate comparison of SCA, IWF, and ZFB with a BS-to-BS separation of $d=5 \mathrm{~km}$

MIMO-OFDM subchannels, which can be obtained by performing SVD. The total number of complex floating point operations is approximately

$$
\sum_{k=1}^{K} \sum_{n=1}^{N} 8 n k\left[8 L_{R}\left(M L_{T}\right)^{2}+9\left(M L_{T}\right)^{3}\right] .
$$

Therefore, the overall computational complexity of ZFB with semi-orthogonal user selection is given by

$$
\mathcal{O}\left\{K N\left[64 L_{R}\left(M L_{T}\right)^{2}+L_{T}^{3}+72\left(M L_{T}\right)^{3}\right]\right\} .
$$

\section{Conclusions}

In this paper, the individual UT rate target is achieved by transforming a non-convex optimization problem into a tractable set of successive convex approximations. A convex lower bound is updated at each iteration to improve the approximation of the achievable rate region, where a dual Lagrange decomposition and a subgradient method is efficient in obtaining the locally optimal solution. Average power constraints are enforced on each antenna for all BSs, which helps manage the resulting peak power effects (via OFDM's inherently high PAPR) for all transmission high-powered amplifiers. We envision this work to be more suited for small cells with low user mobility, but more importantly, for fixed-wireless applications in sparsely populated regions that require high data rates to UTs over very large network areas.

The effectiveness of our proposed SCA-based algorithm was demonstrated through a performance comparison of SCA and the alternative approaches of IWF in [24] and ZFB in [15]. Comparing SCA and IWF, we see that SCA provides a lower total transmit power and higher minimum per-UT rate target relative to IWF in a range of interference environments. In general, we find that the higher the interference between UTs, the larger difference in terms of total transmit power and minimum per-UT target rate between SCA and IWF. As expected, ZFB performs well in high-interference environments as it provides interference-free subchannels for the scheduled UTs. However, the performance of ZFB is limited by the number of transmit antennas and the mutual orthogonality of the scheduled UTs' channel conditions. As such, we find that ZFB results in a higher total transmit power and lower minimum achievable rate solution than SCA and IWF in both medium- and low-interference environments.

\section{Competing interests}

The authors declare that they have no competing interests.

Received: 14 May 2015 Accepted: 28 January 2016

Published online: 09 February 2016

References

1. A Ghosh, R Ratasuk, B Mondal, N Mangalvedhe, T Thomas, LTE-Advanced: next-generation wireless broadband technology. IEEE Wirel. Commun. 17(3), 10-22 (2010)

2. M Sawahashi, Y Kishiyama, A Morimoto, D Nishikawa, M Tanno, Coordinated multipoint transmission/reception techniques for LTE-Advanced. IEEE Wirel. Commun. Mag. 17(3), 26-34 (2010)

3. D Lee, H Seo, B Clerckx, E Hardouin, D Mazzarese, SNK Sayana, Coordinated multiple transmission and reception in LTE-Avanced: deployment scenarios and operational challenges. IEEE Commun. Mag. 50(2), 148-155 (2012) 
4. R Irmer, H Droste, P Marsch, M Grieger, G Fettweis, S Brueck, H-P Mayer, L Thiele, $V$ Jungnickel, Coordinated multipoint: concepts, performance, and field trial results. IEEE Commun. Mag. 49(2), 102-111 (2011)

5. J Lee, Y Kim, H Lee, BL Ng, D Mazzarese, J Liu, W Xiao, Y Zhou, Coordinated multipoint transmission and reception in LTE-Avanced systems. IEEE Commun. Mag. 50(11), 44-50 (2012)

6. S Kaviani, WA Krzymień, in Proc. IEEE Wireless Communications and Networking Conference. Sum rate maximization of MIMO broadcast channels with coordinated of base stations, (Las Vegas, 2008), pp. 1079-1084

7. W Hardjawana, B Vucetic, Y Li, Multi-user cooperative base station systems with joint processing and beamforming. IEEE J. Sel. Topics Signal Process. 3(6), 1079-1093 (2009)

8. R Zhang, Cooperative multi-cell block diagonalization with per-base-station power constraints. IEEE J. Sel. Areas Commun. 28(9), 1435-1445 (2010)

9. CY Hsu, BS Krongold, in Proc. IEEE Global Communications Conference. Coordinated multi-point transmission of MIMO-OFDM system with per-antenna power constraints, (Anaheim, 2012)

10. BS Krongold, KRamchandran, DL Jones, Computationally efficient optimal power allocation algorithm for multicarrier communication systems. IEEE Trans. Commun. 48(1), 23-27 (2000)

11. MHM Costa, Writing on dirty paper. IEEE Trans. Inf. Theory. 29(3), 439-441 (1983)

12. DHN Nguyen, T Le-Ngoc, Sum-rate maximization in the multicell MIMO broadcast channel with interference coordination. IEEE Trans. Signal Process. 62(6), 1501-1513 (2014)

13. W Yu, W Rhee, S Boyd, JM Cioffi, Iterative water-filling for Gaussian vector multiple-access channel. IEEE Trans. Inf. Theory. 50(1), 145-152 (2004)

14. QH Spencer, AL Swindlehurst, M Haardt, Zero-forcing methods for downlink spatial multiplexing in multiuser MIMO channels. IEEE Trans. Signal Process. 52(2), 461-471 (2004)

15. S Kaviani, WA Krzymień, in Proc. IEEE Global Communications Conference. User selection for multiple-antenna broadcast channel with zero-forcing beamforming, (New Orleans, 2008)

16. M Pischella, J-C Belfiore, Distributed resource allocation for rate-constrained users in multi-cell OFDMA networks. IEEE Commun. Lett. 12(4), 250-252 (2008)

17. C Hellings, M Joham, W Utschick, Gradient-based power minimization in MIMO broadcast channels with linear precoding. IEEE Trans. Signal Process. 60(2), 877-890 (2012)

18. J Papandriopoulos, JS Evans, SCALE: A low-complexity distributed protocol for spectrum balance in multiuser DSL networks. IEEE Trans. Inf. Theory. 8(8), 3711-3724 (2009)

19. NU Hassan, M Assaad, in Proc. IEEE International Workshop on Signal Processing Advances in Wireless Communications. Optimal downlink beamforming and resource allocation in MIMO-OFDMA systems, (Marrakech, 2011)

20. L Venturino, N Prasad, X Wang, Coordinated scheduling and power allocation in downlink multicell OFDMA networks. IEEE Trans. Veh. Technol. 6(58), 2835-2848 (2009)

21. H Zhu, J Wang, Chunk-based resource allocation in OFDMA systems-part i: chunk allocation. IEEE Trans. Commun. 57(9), 2734-2744 (2009)

22. H Zhu, J Wang, Chunk-based resource allocation in OFDMA systems - part ii: joint chunk, power and bit allocation. IEEE Trans. Commun. 60(2), 499-509 (2012)

23. S Boyd, L Vandenberghe, Convex Optimization. (Cambridge University Press, Cambridge, 2004)

24. M Kobayashi, G Caire, in Proc. IEEE International Conference on Acoustics, Speech and Signal Processing. Iterative waterfilling for weighted rate sum maximization in MIMO-OFDM broadcast channels, (2007)

25. A Goldsmith, Wireless Communications. (Cambridge University Press, New York, 2005)

26. HR Anderson, Fixed Broadband Wireless System Design. (Wiley, UK, 2003)

27. GL Stüber, J Barry, SW McLaughlin, YG Li, MA Ingram, TG Pratt, Broadband MIMO-OFDM wireless communication. Proc. IEEE. 92, 271-294 (2004)
28. R Horst, H Tuy, Global Optimization: Deterministic Approaches, 2nd edn. (Springer, Berlin, 1993)

29. RD Yates, A framework for uplink power control in cellular radio system. IEEE J. Sel. Areas Commun. 13(7), 1341-1347 (1995)

30. H Holma, A Toskala (eds.), WCDMA for UMTS - HSPA Evolution And LTE, 4th edition (Wiley, UK, 2007) 
31. H Holma, A Toskala (eds.), LTE for UMTS: OFDMA and SC-FDMA Based Radio access (Wiley, UK, 2009)

32. GH Golub, CFV Loan, Matrix Computations. (John Hopkins University Press, Baltimore, 1996)

33. J Mao, J Gao, Y Liu, G Xie, Simplified semi-orthogonal user selection for MU-MIMO systems with ZFBF. IEEE Wirel. Commun. Lett. 1(1), 42-45 (2012)

\section{Submit your manuscript to a SpringerOpen ${ }^{\circ}$ journal and benefit from:}

- Convenient online submission

Rigorous peer review

- Immediate publication on acceptance

- Open access: articles freely available online

High visibility within the field

- Retaining the copyright to your article

Submit your next manuscript at $\mathbf{s p r i n g e r o p e n . c o m ~}$ 